\title{
Quantitative comparison of grasp and release abilities with and without functional neuromuscular stimulation in adolescents with tetraplegia
}

\author{
BT Smith, MJ Mulcahey and RR Betz
}

Research Department, Shriners Hospitals for Crippled Children, 8400 Roosevelt Blvd., Philadelphia, PA 19152, USA

\begin{abstract}
In this study, a functional neuromuscular stimulation (FNS) hand system designed by Case Western Reserve University (CWRU) was implemented in 5 adolescents with C5 tetraplegia and hand function with FNS was evaluated and compared to tenodesis abilities using a grasp and release test designed specifically for this purpose. The test involved the unilateral acquisition, movement and release of 6 objects of various sizes and weights. During a single test session, five 30-second trials were performed with each object with and without the FNS system where the number of completions and failures were recorded for each trial. At least 4 and as many as 8 test sessions were conducted with each subject over periods of 1.5 to 3 years. For each subject, test performance with FNS was compared statistically to tenodesis performance and session-to-session consistency was analyzed. Of 30 comparisons (5 subjects and 6 test objects), FNS was more effective in 23 cases $(77 \%)$, tenodesis was better in 5 instances $(17 \%)$ and in 2 cases $(6 \%)$ there was no difference. Subjects were able to manipulate the 3 heaviest test objects only with FNS and in $60 \%$ of all cases involving the 3 lighter test objects there were significantly more trials in which there were more completions or fewer failures with FNS. Performance with FNS and tenodesis was generally inconsistent across sessions; possible reasons for these variations are discussed. Overall, the results of this study support those of the only previous investigation that used the grasp and release test to evaluate hand function with FNS in C5 and C6 tetraplegia.
\end{abstract}

Keywords: functional neuromuscular stimulation; spinal cord injury; pediatrics; outcome measures

\section{Introduction}

For individuals with mid-cervical level spinal cord injuries (SCI), a small but effective workspace can be realized with residual arm movements, but with the loss of active finger and thumb motion, hand function must be accomplished with a combination of passive grasp and release abilities or tenodesis*, compensatory hand skills developed through therapy, and adaptive equipment. Even with these adaptive mechanisms, object manipulation is often difficult or, in some cases, impossible. For this reason, research efforts employing functional neuromuscular stimulation (FNS) have concentrated primarily on providing active grasp and release function for this group. ${ }^{1}$

While several FNS hand systems have been described in the literature over the past decade, ${ }^{2-8}$ there is little quantitative clinical data about their effectiveness. Evaluations of FNS systems have been

Correspondence: BT Smith

*Tenodesis refers to the ability to generate passive opening and closing of the hand with changes in wrist position. When the wrist is flexed the fingers and thumb extend. Wrist dorsiflexion generates a grasp by passive flexion of the fingers and thumb. For those with mid-cervical SCI, gravity is used to flex the wrist and wrist dorsiflexion is accomplished by passively positioning the hand or volitionally for those with residual C6 motor activity based largely on clinical observations of activities of daily living (ADL), and interviews with system users. ${ }^{6,8,9}$ These are important factors in determining the benefits of this technology. However, without quantitative measures of function one cannot objectively compare FNS systems to one another or to other interventions, assess performance across subjects, or evaluate system enhancements.

The absence of quantitative studies can perhaps be attributed to the lack of a universally accepted test of hand function for the population with tetraplegia. ${ }^{10}$ An extensive review by Stroh and colleagues ${ }^{11}$ revealed several limitations to existing assessments as they relate to tetraplegic hand function including the inability to isolate hand function from arm or trunk movements, the inclusion of complex grasps that are inappropriate for the population with tetraplegia, and subjective evaluation criteria.

Due to these limitations in existing tests of hand function, researchers at CWRU developed two assessments to quantify and compare the functional benefits of FNS for active hand function to traditional rehabilitation options. ${ }^{11,12}$ One test uses a task analysis approach to examine a person's level of independence during ADL. This test also evaluates the users' 
satisfaction with the FNS system. ${ }^{12}$ The second assessment is a grasp and release test that involves the unilateral manipulation of six standardized objects of various sizes and weights. ${ }^{11}$

In collaboration with the Rehabilitation Engineering Center at CWRU, our institution employed both the ADL and grasp and release test to quantify the functional benefits of the CWRU hand system ${ }^{2}$ for five adolescents with C5 tetraplegia. Results of the ADL test have been reported previously. ${ }^{13}$ This paper describes results of the grasp and release test administered to the same five adolescents, comparing FNS hand function to tenodesis abilities.

\section{Methods}

\section{Subjects}

Five subjects from 13 to 19 years of age participated in the study (Table 1). Time since injury ranged from 3 to 72 months. Each adolescent sustained a mid-cervical level spinal cord injury (SCI) and is classified as O:0 or $\mathrm{O}: 1$ according to the international classification for surgery of the hand in tetraplegia. ${ }^{14}$ Total motor index scores ranged from 10 to 15 according to the American Spinal Injury Association guidelines. ${ }^{15}$ Subject 2 underwent surgical transfer of the brachioradialis to the radial wrist extensors in the extremity with FNS which provided grade 2 wrist extension strength. For subjects 3 , 4, and 5 wrist extension strength of the FNS extremity increased from grade 2 to grade 3 by the end of the first year of participation. Although unable to volitionally extend her wrist, subject 1 used gravityassisted dorsiflexion and volar flexion to achieve tenodesis function. After 1 year of participation, subject 1 also underwent surgery to augment stimulation of the hand. ${ }^{16}$ This included a Zancolli lasso intrinsic tenodesis, reefing of the extensor mechanism, synchronization of the FDP tendons, and transfer of the paralyzed flexor carpi ulnaris (FCU) to the flexor digitorum profundus (FDP). The FCU and FDP muscles were both stimulated to provide finger flexion. In addition, an arthrodesis of the distal interphalangeal (IP) joint of the middle finger and IP joint of the thumb were performed.
CWRU hand system

The CWRU system ${ }^{2}$ provided lateral and palmar prehension by stimulation of selected forearm and hand muscles using chronically-indwelling percutaneous electrodes. A microprocessor-controlled stimulator unit coordinated excitation of the muscles based on two control inputs. A small switch mounted on the chest allowed the user to select between lateral and palmar grasp patterns and turn the system on or off. A position transducer mounted across the shoulder joint opposite the instrumented hand, translated relative shoulder position in the elevation/depression axis to a proportional signal that controlled opening and closing of the hand. The relationship between relative shoulder position and the stimulus levels of different muscle groups was derived from grasp templates established by Kilgore et al. ${ }^{17}$ Maximum grasp extension corresponded to a neutral or 'resting' shoulder position. With elevation of the shoulder from that position, finger and thumb flexion was graded such that maximum force was accomplished in roughly half of the active range of shoulder motion. Shoulder depression reversed the process, opening the hand. For the lateral pinch, flexion of the thumb and fingers was coordinated so that a pinch was created between the thumb and lateral aspect of the index finger. For palmar grasp, the thumb was stimulated constantly in abduction and shoulder motion regulated extension and flexion of the fingers. ${ }^{17}$

\section{Grasp and release test implementation}

Each subject underwent percutaneous intramuscular electrode implantations, ${ }^{18}$ stimulated exercise to reverse disuse atrophy, and training in system operation, grasp coordination, and ADL use. ${ }^{13}$ Following training, the first grasp and release test was conducted. Subsequent tests were administered during follow-up visits which spanned 1.5 to three years and included at least four and as many as eight tests. All tests were conducted by the same therapist (MJM) who was trained in its administration.

The grasp and release test procedures are summarized here and described in detail elsewhere. ${ }^{11}$ The test involved the manipulation of six objects representing a

Table 1 Clinical summary of the five participants

\begin{tabular}{|c|c|c|c|c|c|c|c|c|}
\hline \multirow[b]{2}{*}{ Subject } & \multirow[b]{2}{*}{$A g e^{*}$} & \multirow[b]{2}{*}{ Sex } & \multirow[b]{2}{*}{$L O I^{*}$} & \multirow{2}{*}{$\begin{array}{c}\text { Time } \\
\text { post- } \\
\text { injury* } \\
\text { (months) }\end{array}$} & \multirow{2}{*}{$\begin{array}{l}\text { International } \\
\text { hand } \\
\text { classification }\end{array}$} & \multirow{2}{*}{$\begin{array}{l}\text { Total } \\
\text { motor } \\
\text { index* }\end{array}$} & \multicolumn{2}{|c|}{$\begin{array}{l}\text { Wrist extension strength } \\
\text { (extremity with FNS) }\end{array}$} \\
\hline & & & & & & & $\underset{1^{*}}{\text { Measurement }}$ & $\begin{array}{c}\text { Measurement } \\
2+\end{array}$ \\
\hline 1 & 18 & $\mathrm{~F}$ & C5 & 61 & O:0 & 10 & 0 & 0 \\
\hline 2 & 19 & $\mathbf{M}$ & $\mathrm{C} 5$ & 72 & $\mathrm{O}: 1$ & 10 & 0 & $2 * *$ \\
\hline 3 & 17 & $\mathbf{M}$ & $\mathrm{C} 5$ & 5 & O:1 & 15 & 2 & 3 \\
\hline 4 & 13 & $\mathrm{~F}$ & $\mathrm{C} 5$ & 8 & O:1 & 14 & 2 & 3 \\
\hline 5 & 13 & $\mathbf{M}$ & $\mathrm{C} 5$ & 3 & $\mathrm{O}: 1$ & 15 & 2 & 3 \\
\hline
\end{tabular}


Table 2 Grasp and release test objects

\begin{tabular}{lccc} 
Test object & Grasp & $\begin{array}{c}\text { Object } \\
\text { weight }(N)\end{array}$ & $\begin{array}{c}\text { Object size } \\
(\mathrm{cm})\end{array}$ \\
\hline Peg & Lateral & 0.016 & $7.6 \times 0.6$ (diameter) \\
Block & Palmar & 0.104 & $2.5 \times 2.5 \times 2.5$ \\
Can & Palmar & 2.1 & $9.1 \times 2.4$ (diameter) \\
Weight & Lateral & 2.59 & $5 \times 1.4$ (thickness) \\
Videotape & Palmar & 3.49 & $20.4 \times 12.0 \times 3.0$ \\
Fork & Lateral & $4.4^{*}$ & $14.5 \times 1.2$ (diameter) \\
\hline * Vertical force required to depress cylinder against spring
\end{tabular}

range of weights and sizes (Table 2). Each test object is intended to be analogous to one or more objects encountered during ADL. Three objects were grasped with the lateral pinch (peg, weight, fork) and three with the palmar grasp (block, can, tape). Object manipulation was structured in the following way. The 'fork', a cylindrical handle connected to a springloaded piston, was grasped, depressed $2 \mathrm{~cm}$ against the force of the spring (requiring $4.4 \mathrm{~N}$ of vertical force) and released. The other 5 objects were acquired lateral to midline, carried over a $4.5 \mathrm{~cm}$ barrier and released at midline. A successful completion required that the object be acquired between the thumb and index finger (lateral pinch) or the thumb and four fingers (palmar grasp), carried without being dropped or using gravity as an assist, and placed in an upright position upon release. $^{11}$

At the start of each session, a pretest was conducted in which the manipulation of each object was attempted with and without FNS. The purpose of the pretest was to determine whether the subject understood the instructions, whether each object could be manipulated with and without FNS, and to provide an opportunity for the subject to develop a strategy for manipulation. During the pretest, if a test object could not be manipulated at least once according to the test instructions, then it was excluded from the main test for that method of prehension. For example, if the weight task could be completed only with FNS on the pretest, then it was tested only using FNS during the main test.

Following the pretest, five 30 -second trials were conducted for each object and prehension method combination that was successfully completed during the pretest. Objects were presented in random order using a minimum 30 -second rest period between trials. During each trial, the subject was asked to complete as many tasks as possible within the allotted time according to the standardized instructions. It was emphasized that equal weight would be given to maximizing completions and minimizing failures. The number of completions and failures was immediately tabulated. ${ }^{11}$

For each testing session, subjects were positioned in their wheelchair with their upper extremity in an identical position relative to the objects. The height of the test table, the position of the test board with respect to the edge of the table and the distance between the wheelchair and table were established during the first pretest and remained unchanged thereafter.

Before the main test, 5 grasp force measurements of lateral and palmar prehension were taken using a modified pinchmeter. For these measurements the arm was adducted, the elbow was at $90^{\circ}$ degrees flexion and the forearm was in a neutral position. While measuring tenodesis flexion, wrist extension was encouraged. When using FNS, three subjects wore a dorsal splint to stabilize the wrist and two subjects (1 and 4) wore orthotics fabricated specifically to provide wrist stability and prevent metacarpophalangeal (MCP) hyperextension during stimulated extension. Without FNS, no splints were worn and each subject used tenodesis grasp and release.

\section{Data analysis}

Two hypotheses were tested. The first hypothesis was that there would be no difference in performance between FNS and tenodesis abilities. This was evaluated using a two-tailed paired sign test $(P<0.05)$. For each object-subject combination, the number of paired trials for which there were more completions or failures with FNS were summed and the two-tailed probability of obtaining that number of trials or more was calculated. This resulted in 30 comparisons between FNS and tenodesis performance, 6 object comparisons for each of 5 subjects.

The second hypothesis was that each subject's performance with and without FNS would be consistent across sessions; this was determined using the Kruskall-Wallis statistic $(P<0.05)$. When cases of inconsistent performance appeared by visual analysis to have improved (i.e. increase in median session completions or decrease in median failures), an iterative process was employed to determine if consistency was achieved in later sessions. The Kruskall-Wallis test was applied across all sessions subsequent to the first, then all sessions subsequent to the second, and so on until a plateau in performance was found. If a plateau was found, then those later sessions were collectively compared to the initial session using the Wilcoxon statistic $(P<0.05)$ to determine whether improvements were significant. New tests were included in the analyses except the first three sessions of subject 1 which were excluded due to testing errors.

\section{Results}

\section{FNS vs tenodesis}

Table 3 summarizes FNS and tenodesis pretest performance for each subject. It shows the number of sessions for which each task could be completed and the total percentage of all sessions for which that 
Table 3 Summary of (A) FNS and (B) tenodesis pretest performance. For each subject the total number of sessions and the number of sessions for which each object was successfully manipulated with and without FNS are shown. For each object the total proportion of sessions for which the pretest was passed is also shown across subjects

\begin{tabular}{|c|c|c|c|c|c|c|c|}
\hline \multicolumn{8}{|c|}{ A. FNS pretest performance } \\
\hline Subject & $\begin{array}{c}\text { IOtal } \\
\text { sessions }\end{array}$ & Peg & Block & Can & $W g t$ & Tape & Fork \\
\hline 1 & 5 & A & A & 2 & 4 & $1^{+}$ & 4 \\
\hline 2 & 8 & A & A & 7 & A & 6 & A \\
\hline 3 & 7 & A & A & A & A & A & 6 \\
\hline 4 & 4 & A & $A^{*}$ & $A^{*}$ & A & $\dagger^{*}$ & 3 \\
\hline 5 & 4 & A & A & A & 3 & 2 & 2 \\
\hline Total & 28 & $100 \%$ & $100 \%$ & $86 \%$ & $93 \%$ & $57 \%$ & $82 \%$ \\
\hline \multicolumn{8}{|c|}{ B. Tenodesis pretest performance } \\
\hline Subject & $\begin{array}{c}\text { Total } \\
\text { sessions }\end{array}$ & Peg & Block & Can & $W g t$ & Tape & Fork \\
\hline 1 & 5 & 2 & $\dagger$ & $\dagger$ & $\dagger$ & $\dagger$ & $\dagger$ \\
\hline 2 & 8 & A & A & A & $\dagger$ & $\dagger$ & $\dagger$ \\
\hline 3 & 7 & A & A & A & $\dagger$ & $\dagger$ & $\dagger$ \\
\hline 4 & 4 & A & $A^{*}$ & $\mathrm{~A}^{*}$ & $\dagger$ & $\dagger^{*}$ & $\dagger$ \\
\hline 5 & 4 & 3 & 3 & $\dagger$ & $\dagger$ & $\dagger$ & $\dagger$ \\
\hline Total & 28 & $86 \%$ & $79 \%$ & $68 \%$ & $0 \%$ & $0 \%$ & $0 \%$ \\
\hline
\end{tabular}

Cells with an 'A' denote instances where the pretest was passed in all sessions and cells with ' $\dagger$ ' indicate when the pretest was never passed. * Tasks were tested in first three sessions only. + Could only be accomplished after hand surgery

object could be manipulated. With FNS, subjects were able to manipulate each test object in at least 1 test session with the exception of subject 4 who could never complete the tape task. With a tenodesis, all subjects were able to complete the peg task, 1 subject could not manipulate the block, 2 subjects could never complete the can task and no subject was able to pass the pretest with the weight, fork or tape.

Figure 1 shows the results of the paired sign test for completions (la) and failures (lb) for each subject and Figure 2 summarizes these results by test object. For 23 of the $30(77 \%)$ task comparisons, performance was significantly improved with FNS. In 14 of the 15 cases involving the heaviest test objects (weight, fork, tape), tasks could only be completed with FNS. For the lighter test objects (peg, block, can), FNS was more effective in 9 of 15 cases $(60 \%)$. In 3 instances ( 2 can, 1 block) FNS was needed to complete the task; 2 of these situations involved subject 1 , the only individual who lacked wrist extension. In 4 instances ( 2 can, 1 block, 1 peg) there was no difference in completions but significantly more trials where there were fewer failures using FNS. In 2 cases ( 1 block, 1 peg) there were more completions with FNS in a greater number of trials. Of the 6 remaining cases with the lighter objects, there was 1 case (can) in which there were no differences in completions or failures and 5 situations (3 peg, 2 block) in which more completions were accomplished using a tenodesis. In 3 of those cases there were also more tenodesis failures.

Table 4 shows the average session grasp forces with FNS. Lateral pinch forces ranged from $8.9 \mathrm{~N}$ to $22.5 \mathrm{~N}$ and palmar grasp forces from $2.1 \mathrm{~N}$ to $11.1 \mathrm{~N}$. Tenodesis grasp force was not measurable.
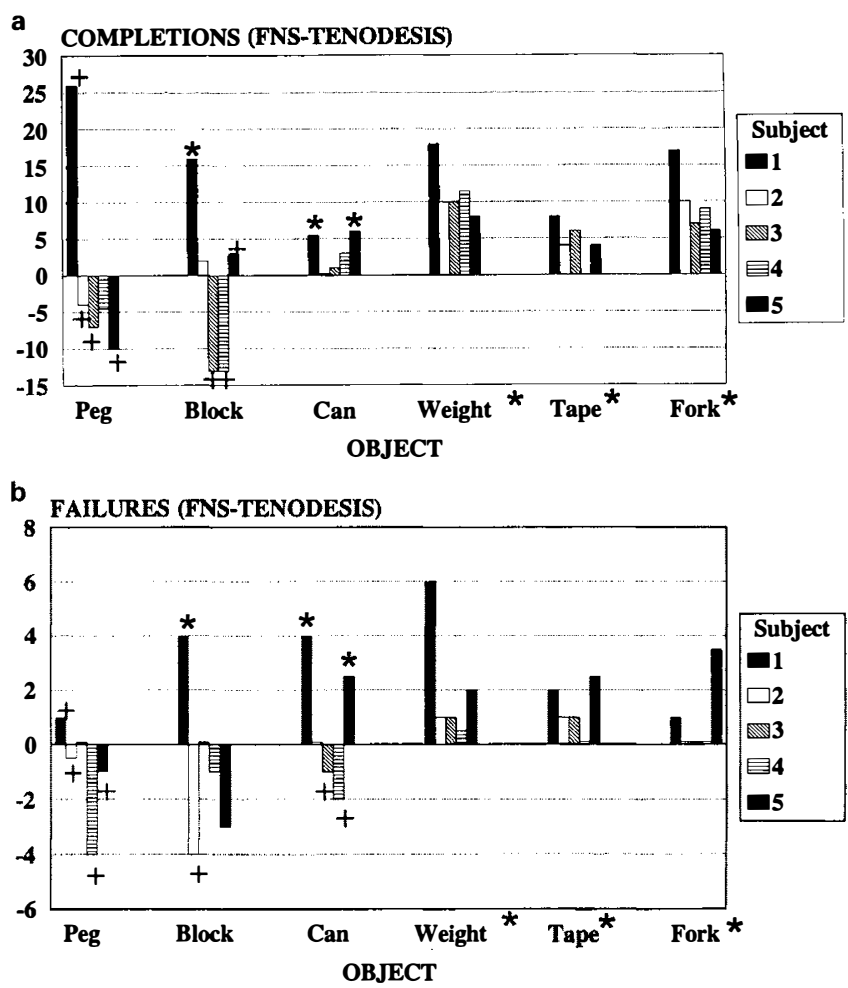

Figure 1 The differences are shown between FNS and tenodesis in the overall median for (a) completions and (b) failures by object and subject. Positive medians indicate when more completions or failures were accomplished with FNS. An asterisk indicates when a task could be completed only with FNS and in these cases the median values for FNS completions and failures are shown. A plus sign represents significant differences across all sessions by the paired sign test $(P<0.05)$ 


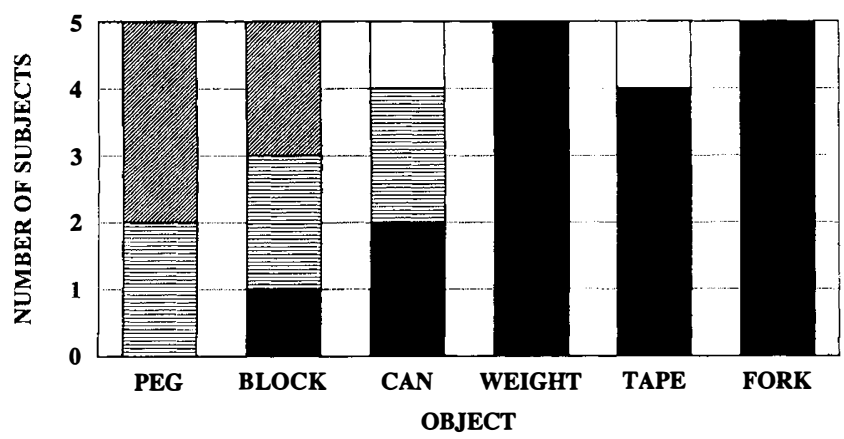

FNS only GFNS better $\square$ No Difference שaT Tenodesis better

Figure 2 Results of the paired sign test for each test object listed from lightest (peg) to heaviest (fork). The graph shows the number of subjects that required FNS (FNS only), those for whom there were a significantly greater number of trials in which there were more completions and/or fewer failures with FNS (FNS better) or with tenodesis (tenodesis better), and situations in which there was no difference between the two (no difference). The weight, tape, and fork could only be manipulated with FNS

Table 4 Average lateral and palmar grasp forces with FNS calculated over all sessions. Five grasp force measures were taken prior to each grasp and release test. For all subjects, tenodesis grasp force was not measureable

\begin{tabular}{lccc}
\hline Subject & $\begin{array}{c}\text { Number of } \\
\text { sessions }\end{array}$ & $\begin{array}{c}\text { Lateral pinch } \\
\text { force }(N)\end{array}$ & $\begin{array}{c}\text { Palmar grasp } \\
\text { force }(N)\end{array}$ \\
\hline 1 & 5 & $10.3 \pm 7.1$ & $2.1 \pm 2.9$ \\
2 & 7 & $13.6 \pm 4.5$ & $11.1 \pm 6.0$ \\
3 & 5 & $22.5 \pm 8.6$ & $9.5 \pm 2.0$ \\
4 & 4 & $12.3 \pm 5.2$ & $5.2 \pm 1.0$ \\
5 & 4 & $8.9 \pm 5.2$ & $6.8 \pm 3.3$ \\
\hline
\end{tabular}

\section{Consistency across sessions}

Of 29 testable cases with FNS, completions were consistent across sessions in 8 instances $(28 \%) ; 6$ of which involved the peg or block. The number of failures was consistent in 10 instances $(34 \%)$. Tenodesis performance was consistent in 3 of the $12(25 \%)$ testable cases for completions and 7 of $12(58 \%)$ instances for failures.

With FNS, 5 of the $21(24 \%)$ inconsistent cases were due to increases in completions in early sessions. In 4 of those cases, the median number of completions plateaued by the second or third session. In the other instance, subject 1 was only able to complete the tape task in the eighth session, after surgery to facilitate stimulated finger extension. With tenodesis, 7 of the 9 $(78 \%)$ inconsistent cases were related to improved performance, all on peg or block tasks. Plateaus in performance occurred anywhere from the second to fourth session. With FNS and tenodesis, each case of improved performance in later sessions was significantly better as compared to the initial session (Wilcoxon, $P<0.05$ ).

\section{Discussion}

In this study, a grasp and release test was administered over periods of 1.5 to 3 years to five adolescents with C5 tetraplegia to quantitatively compare hand function provided by the CWRU FNS hand system to tenodesis abilities. While there are no validity and reliability studies of the grasp and release test, it was developed after a thorough examination of the literature on hand assessments and was designed specifically to quantify grasp and release performance with and without the hand system for the population with C5 or C6 function; this suggests face validity. Further, the test objects required gross manipulation skills appropriate to this population ${ }^{11}$ which suggests content validity.

Results of the test showed that in 23 of the 30 comparisons grasp and release abilities were better with FNS. The FNS system provided the ability to manipulate the heaviest test objects (weight, tape, fork) which could not be done with tenodesis. In addition, in 9 of the 15 comparisons involving the lighter objects (peg, block, can), FNS performance was better; either tasks could be completed only with FNS or there was a significant number of trials in which there were more completions or fewer manipulation errors with the hand system.

In 5 of the 30 comparisons, all involving the peg or block, tenodesis was better than FNS. This involved the subjects with voluntary wrist extension and it appeared that these subjects could manipulate the small, light objects more quickly with tenodesis as compared to operating the FNS grasp with contralateral shoulder movement. However, in those cases tenodesis was not always as accurate: in 3 of the 5 cases there were a significant number of trials in which there were fewer errors using FNS.

While the grasp and release test does not predict the ability to perform ADL with tenodesis or FNS or how either grasping method would be employed in the community, the test results do demonstrate clearly that FNS provided a potentially important capability over tenodesis function. The FNS system allowed all subjects to grasp, move and release the heaviest test objects with one hand unassisted by gravity which could not be accomplished with a tenodesis grasp. For those with $\mathrm{C} 5$ or C6 function, successful manipulation of analogous objects encountered during ADL (lifting a book, picking up a soda can, stabbing with a fork, etc.) is typically accomplished with two hands which greatly challenges sitting balance, limits the workspace, and hinders bilateral activities. Objects that must be maintained in an upright position such as a cup, provide added difficulty. By potentially turning these activities into one handed efforts, FNS may address these deficits. In a previous study, ADL tests 
with these same subjects showed that the FNS system can address these shortcomings and was generally preferred over their alternate methods. In addition, usage surveys indicated that the system was employed successfully in home and school to perform ADL but that the ability to use it on a regular basis relied on adequate family and environmental supports. ${ }^{13}$

The only other studies that employed the grasp and release test to evaluate and compare FNS hand function to tenodesis abilities yielded similar results. ${ }^{11,19}$ Stroh et al. administered the assessment once a week for four weeks to four adults with C5 or C6 tetraplegia fitted with the CWRU hand system. Applying similar analyses, they reported that all subjects could manipulate the weight, tape and fork only with FNS. For the lightest objects (peg, block) FNS was better for those with $\mathrm{C} 5$ function and tenodesis was more effective for those with C6 function. ${ }^{11}$ In another study, the CWRU system was adapted for an eight year old child with a C7 level injury so that the child coordinated stimulated finger and thumb flexion with volitional finger and thumb extension. ${ }^{19}$ Over a 1 year period, five grasp and release tests were administered. The child demonstrated the ability to lift the 3 heaviest objects only with FNS. Hand grasp with FNS was more effective for the can task and tenodesis function was better for the peg and block.

In this study, there was considerable variability in FNS test performance. On several occasions flexion force was not sufficient to pass the pretest with the heavier test objects (Table 3 ) and fatigue appeared to compromise performance during some sessions. One reason for this may have been fluctuations in grasp force (Table 4) and endurance secondary to subjects' irregular participation in stimulated exercise routines. Each subject was asked to exercise with FNS for 6 h, 4 times a week at night in order to maintain muscle strength and endurance, however telephone interviews revealed that exercise time was usually less and varied considerably from week to week. ${ }^{13}$

It was often necessary to replace or reprogram the percutaneous electrodes ${ }^{18}$ between test sessions which likely played a role in variability. However, all attempts were made to provide consistent stimulated grasps. Before each test, grasp modifications were made using rule-based electrode selection criteria and grasp templates ${ }^{17}$ which provided a standardized format to incorporate new electrodes or compensate for changes in recruitment properties unrelated to conditioning such as threshold and maximum stimulation pulse durations.

Another important factor affecting FNS test performance was the ability to achieve adequate finger extension with stimulation of the extensor digitorum communis (EDC). Stimulation of the EDC often resulted in an intrinsic minus posture of the hand and, in combination with flexor tendon tightness, interfered with acquisition of the tape and can, objects that required large hand opening. Three subjects wore an orthosis to block MCP hyperextension and underwent stimulated exercise routines that provided a prolonged stretch of the flexor tendons to reduce tightness. Orthotic management effectively prevented intrinsic-minus posturing, however, even with the exercise routine, flexor tendon tightness impeded extension of the proximal IP joints making it difficult to surround the tape or can and subsequently maintain hold of them. After 1 year of orthotic use, subject 1 underwent a Zancolli lasso procedure to augment stimulated finger extension. ${ }^{16}$ This procedure provided an internal block of the MCP joints and, by transfer of the flexor digitorum superficialis, may have reduced the effects of flexor tendon tightness at the proximal IP joints. After surgery, she was able to use FNS to successfully acquire, maintain hold of and release the tape, which could not be accomplished with FNS using the orthosis. The Zancolli lasso intrinsic tenodesis is now considered an important and effective adjunct to the CWRU hand system and is performed with implementation of their implantable system. ${ }^{5}$ Stimulation of the finger intrinsics may be another way to improve finger extension. Promising preliminary work has been reported, ${ }^{20-22}$ however stimulation of intrinsic muscles is not yet a standard component to any existing hand system.

Improvements in FNS test performance during early sessions also contributed to variability. In these cases, performance stabilized by the second or third session, approximately 6 months into the program. It was evident that learning to control and use stimulated grasps continued after initial training sessions. This was facilitated by additional training provided at follow-up admissions and experience gained with use of the FNS system at home.

Tenodesis performance was also variable. However, 7 of the 9 inconsistent cases for completions were due to early increases on peg and block tasks involving subjects $2-5$. Improvements appeared to be related to improved tenodesis abilities. For each of the three subjects $(3,4,5)$ injured less than 1 year, natural recovery of wrist extension strength to grade 3 was measured (Table 1). Subject 2 developed wrist extension strength to grade 2 as a result of a brachioradialis tendon transfer to the radial wrist extensors. Importantly, during their early stages of FNS participation these subjects were also involved in rehabilitation sessions designed to facilitate tenodesis use for ADL.

Whether the grasp and release test is sensitive to changes in tenodesis function has not been established. However, Stroh and colleagues have suggested extension of the test to measure performance before and after reconstructive hand surgery or with and without interventions such as the wrist-driven flexor hinge splint. ${ }^{11}$ In addition, the results of a pilot study designed to evaluate tendon transfer surgery for active grasp in a child with a C7 SCI showed that all six test objects were sensitive to the surgical intervention. ${ }^{23}$ 
Using a single subject design, multiple grasp and release tests were administered prior to surgery and at regular intervals within the first year after surgery. The 3 heaviest test objects could be manipulated only after surgery and there were significantly more median completions for the other objects.

Practice effects, which could also account for improved performance, were not apparent in this study and were not reported in the other investigations. ${ }^{11,19,23}$ The test employed standardized objects and rigid test instructions which left little latitude for subjects to deviate from the intended method of task completion. In addition, before each test session, subjects were given time to develop strategies to manipulate objects with FNS and tenodesis.

It could not be determined whether chronic stimulation of the finger and thumb extensor and flexors affected test performance with the tenodesis. Subjects were undergoing changes in tenodesis abilities unrelated to stimulation and there were no tests administered prior to the implementation of FNS which prevented an examination of crossover effects. Theoretically, the exercise program employed with several subjects to reduce flexor tendon tightness could have acted to decrease tenodesis flexion force and increase tenodesis opening, however, the effectiveness of this conditioning routine was not evaluated.

Although the assessment was designed for adults, it appeared to be suitable for use with the youngest adolescents as there were no apparent age-related differences in performance (Figure 1). The two 13 year old subjects appeared to fully understand test instructions and tolerate the duration of the test (about $1.5 \mathrm{~h}$ ). In addition, the size and weight of test objects seemed to be appropriate. Our research laboratory has also applied the test successfully to an eight year old child. ${ }^{19}$ These preliminary applications of the grasp and release test with children are encouraging and further investigation of the test's applicability to the pediatric with population SCI is warranted.

\section{Conclusion}

The CWRU percutaneous FNS hand system was implemented in 5 adolescents with $\mathrm{C} 5$ tetraplegia and CWRU's grasp and release test was administered over periods of 1.5 to 3 years. According to the test, all subjects' unilateral grasp and release abilities were significantly improved with FNS as compared to tenodesis function. Subjects were able to grasp, move and release the three heaviest test objects with FNS only and, in more than half the cases with the lighter, smaller objects, FNS was more effective. FNS test performance was variable from session-to-session which appeared to be related to fluctuations in grasp strength and endurance secondary to muscle conditioning, difficulties achieving adequate stimulated finger extension, and improvements in some subjects' ability to use FNS. Tenodesis test performance was also variable but was related primarily to improved manipulation of the lightest test objects. This appeared to be due to enhanced tenodesis abilities related to natural recovery of wrist extension strength for the acutely injured subjects and tendon transfer surgery for active wrist extension in another subject.

\section{Acknowledgements}

The authors acknowledge Ronald $\mathbf{J}$ Triolo for his assistance in obtaining funding for this investigation. Also acknowledged are the Rehabilitation Engineering Center (REC) staff at Case Western Reserve University (CWRU), Cleveland, $\mathrm{OH}$ for their technical support and advice. Support of the National Institute on Disability and Rehabilitation Research (NIDRR) Grant No. H133E80020 to the CWRU REC provided staff support at CWRU and all patient equipment and electrodes for the study. The time and effort of the adolescents and the support of their families are greatly appreciated. This research was supported by Shriners Hospitals for Crippled Children Grant no. 15954

\section{References}

1 Yarkony GM, Roth EJ, Cybulski G, Jaeger RJ. Neuromuscular stimulation in spinal cord injury $\mathrm{I}$ : restoration of functional movement of the extremeties. Arch Phys Med Rehabil 1992; 73: $78-86$.

2 Buckett JR, Braswell S, Peckham PH, Thrope GB, Keith MW. A portable functional neuromuscular stimulation system. Proceedings IEEE Seventh Annual Conference EMBS 1985; 314-317.

3 Smith B, Peckham PH, Keith MW, Roscoe DD. An externally powered, multichannel, implantable stimulator for versatile control of paralyzed muscle. IEEE Trans Biomed Eng 1987; 34: $499-508$

4 Handa Y, Handa T, Hoshimiya N. A portable FNS system for the paralyzed upper extremities. Proceedings IEEE Eighth Annual Conference EMBS: 1986; 658-660.

5 Keith MW, Peckham PH, Thrope GB, Stroh KC, Smith B, Buckett JR, Kilgore KL, Jatich W. Implantable functional neuromuscular stimulation in the tetraplegic hand. J Hand Surg 1989; 14A: $524-530$

6 Rudel D, Bajd T, Rebersek S, Vodovnik L. FES-assisted manipulation in quadriplegic patients. 7th Int Symp on External Control of Human Extremities: 1981; 273-282.

7 Kiwerski J, Weiss M, Pasniczek R. Electrostimulation of the median nerve in tetraplegics by means of implanted stimulators. Paraplegia 1983; 21: $322-326$

8 Perkins TA, Brindley GS, Donaldson N, Polkey CE, Rushton DN. Implant provision of key, pinch and power grips in a C6 tetraplegic. Med Biol Eng Comp 1994; 32: 367-372.

9 Wijman CA, Stroh KC, Van Doren CL, Thrope GB, Peckham $\mathrm{PH}$, Keith MW. Functional evaluation of quadriplegic patients using a hand neuroprosthesis. Arch Phys Med Rehabil 1990; 71: $1053-1057$

10 Gorman PH, Peckham PH. Upper extremity functional neuromuscular stimulation. J Neuro Rehab 1991; 5: 3-11.

11 Stroh Wuolle KC, Van Doren CL, Thrope GB, Keith MW, Peckham PH. Development of a quantitative hand grasp and release test for patients with tetraplegia using a hand neuroprosthesis. J Hand Surg 1994; 19A: 209-218.

12 Stroh K, Van Doren C, Thrope G, Wijman C. Common object test: A functional assessment for quadriplegic patients using an FNS hand system. Proceedings of the RENSA 12th Annual Conference: $1989 ; 387-388$. 
13 Mulcahey MJ, Smith BT, Betz RR, Triolo RJ, Peckham PH Functional neuromuscular stimulation: functional outcomes in young people with tetraplegia. J Amer Para Soc 1994; 17: 20 - 35

14 McDowell CL, Moberg EA, House JH. The second international conference on surgical rehabilitation of the upper limb in Tetraplegia (Quadriplegia). J Hand Surg 1986; 11A: 604-608.

15 American Spinal Injury Association. Standards for neurological and functional classification of spinal cord injury. Atlanta, Georgia: American Spinal Injury Association 1992.

16 Mulcahey MJ, Betz RR, Smith BT, Triolo RJ, Weiss AA, Keith MW. Functional neuromuscular stimulation and surgical reconstruction of the hand in long-term tetraplegia. J Amer Para Soc 1993; 16: 133.

17 Kilgore KL, Peckham PH, Thrope GB, Keith MW, GallagherStone KA. Synthesis of hand grasp using functional neuromuscular stimulation. IEEE Trans Biomed Eng 1989; 36: 761 - 770.

18 Smith BT, Betz RR, Mulcahey MJ, Triolo RJ. Reliability of percutaneous intramuscular electrodes for upper extremity functional neuromuscular stimulation in adolescents with C5 tetraplegia. Arch Phys Med Rehabil 1994; 75: 939 - 945.
19 Smith BT, Mulcahey MJ, Triolo RJ, Betz RR. The application of a modified neuroprosthetic hand system in a child with a $C 7$ spinal cord injury. Case report. Paraplegia 1992; 30: 598-606.

20 Hendrix LA, Mansour JM. Functional grasp potential of the intrinsic minus hand. IEEE Trans Rehab Eng 1993; 1: 145-153.

21 Fukamachi H, Handa Y, Naito A, Ichie M, Yajima M, Ushikoshi $\mathrm{K}$, Tuchiya M, Matsuchita N, Hoshiyama N. Improvement of finger movement by intrinsic muscles stimulation of the hand. 1987; Proceedings IEEE Ninth Annual Conference EMBS: 361 362.

22 Lauer RT, Kilgore KL, Peckham PH. Control of the hand intrinsic muscles by functional electrical stimulation. 1995; Proceedings of the RENSA '95 Annual Conference: 384-386.

23 Mulcahey MJ, Smith BT, Betz RR, Weiss AA. Outcomes of tendon transfer surgery and occupational therapy in a child with tetraplegia secondary to spinal cord injury. Amer J Occ Ther 1995; 49(7): $607-617$. 